PROCEEDINGS OF THE

AMERICAN MATHEMATICAL SOCIETY

Volume 129, Number 8, Pages 2359-2363

S 0002-9939(01)06009-9

Article electronically published on January 18, 2001

\title{
AN EXAMPLE ON THE MANN ITERATION METHOD FOR LIPSCHITZ PSEUDOCONTRACTIONS
}

\author{
C. E. CHIDUME AND S. A. MUTANGADURA
}

(Communicated by Jonathan M. Borwein)

\begin{abstract}
An example of a Lipschitz pseudocontractive map with a unique fixed point is constructed for which the Mann iteration sequence fails to converge. This resolves a long standing open problem.
\end{abstract}

\section{INTRODUCTION}

Let $K$ be a nonempty closed convex and bounded subset of a real uniformly convex Banach space and $T: K \rightarrow K$ be a nonexpansive mapping (i.e. $\|T x-T y\| \leq$ $\|x-y\|$ for all $x, y \in K$ ). Then $T$ has a fixed point $x^{*} \in K$ (see, e.g., [1, 5, 8]). Unlike in the case of the Banach contraction mapping principle, trivial examples show that the sequence of successive approximations $x_{n+1}=T x_{n}, x_{0} \in K, n \geq 0$, for a nonexpansive map $T$ even with a unique fixed point may fail to converge to the fixed point. It suffices, for example, to take for $T$, a rotation of the unit ball in the plane around the origin of coordinates (see, e.g., [10]). Krasnoselski [9, however, has shown that in this example, one can obtain a convergent sequence of successive approximations if instead of $T$ one takes the auxiliary nonexpansive mapping $\frac{1}{2}(I+T)$, where $I$ denotes the identity transformation of the plane, i.e., if the sequence of successive approximations is defined, for arbitrary $x_{0} \in K$, by

$$
x_{n+1}=\frac{1}{2}\left(x_{n}+T x_{n}\right), n \geq 0,
$$

instead of by the usual so-called Picard iterates, $x_{n+1}=T x_{n}, x_{0} \in K, n \geq 0$. It is easy to see that the mappings $T$ and $\frac{1}{2}(I+T)$ have the same set of fixed points, so that the limit of a convergent sequence defined by (1.1) is necessarily a fixed point of $T$.

More generally, if $E$ is a normed linear space and $K$ is a convex subset of $E$, a generalization of (1.1) which has proved successful in the approximation of fixed points of nonexpansive mappings $T: K \rightarrow K$ (when they exist) is the following scheme (Schaefer [12]):

$$
x_{0} \in K, x_{n+1}=(1-\lambda) x_{n}+\lambda T x_{n}, n \geq 0, \lambda \in(0,1) .
$$

However, the most general iterative scheme now studied is the following:

$$
x_{0} \in K, x_{n+1}=\left(1-c_{n}\right) x_{n}+c_{n} T x_{n}, n \geq 0,
$$

Received by the editors December 14, 1999.

2000 Mathematics Subject Classification. Primary 47H09, 47J25.

(C)2001 American Mathematical Society 
where $\left\{c_{n}\right\} \subset(0,1)$ is a real sequence satisfying appropriate conditions. Under the following additional assumptions: (i) $\lim c_{n}=0$; (ii) $\sum_{n=0}^{\infty} c_{n}=\infty$, the sequence $\left\{x_{n}\right\}$ generated from (1.2) is generally referred to as the Mann sequence in light of [10]. The recursion formula (1.2) has also been used to approximate solutions of numerous nonlinear operator equations and nonlinear variational inclusions in Banach spaces (see, e.g., [3, 4, 6, 11]). A class of nonlinear mappings more general than and including the nonexpansive mappings is the class of pseudocontractions. A mapping $T$ with domain $D(T)$ and range $R(T)$ in $E$ is called pseudocontractive if $\langle T x-T y, j(x-y)\rangle \leq\|x-y\|^{2}$ for each $x, y \in D(T)$ and some $j(x-y) \in J(x-y)$ where $J: E \rightarrow 2^{E^{*}}$ is the normalized duality mapping (see, e.g., [4]). If $K$ is a compact convex subset of a Hilbert space, and $T: K \rightarrow K$ is Lipschitz and pseudocontractive, then by the Schauder fixed point theorem, $T$ has a fixed point in $K$. All efforts to approximate such a fixed point by means of the Mann iteration sequence proved abortive.

In 1974, Ishikawa introduced a new iteration scheme (defined below) and proved the following theorem.

Theorem 1.1 ([7]). If $K$ is a compact convex subset of a Hilbert space $H, T$ : $K \mapsto K$ is a Lipschitzian pseudocontractive map and $x_{0}$ is any point of $K$, then the sequence $\left\{x_{n}\right\}_{n \geq 0}$ converges strongly to a fixed point of $T$, where $x_{n}$ is defined iteratively for each positive integers $n \geq 0$ by

$$
\begin{aligned}
x_{n+1} & =\left(1-\alpha_{n}\right) x_{n}+\alpha_{n} T y_{n}, \\
y_{n} & =\left(1-\beta_{n}\right) x_{n}+\beta_{n} T x_{n},
\end{aligned}
$$

where $\left\{\alpha_{n}\right\},\left\{\beta_{n}\right\}$ are sequences of real numbers satisfying the conditions (i) $0 \leq$ $\alpha_{n} \leq \beta_{n}<1$; (ii) $\lim _{n \rightarrow \infty} \beta_{n}=0$; (iii) $\sum_{n \geq 0} \alpha_{n} \beta_{n}=\infty$.

Since its publication in 1974, it has remained an open question (see, e.g., 6] of whether or not the Mann recursion formula defined by (1.2), which is certainly simpler than the Ishikawa recursion formula (1.3), converges under the setting of Theorem 1.1 to a fixed point of $T$ if the operator $T$ is pseudocontractive and continuous (or even Lipschitzian with constant $L>1$ ). In [2, Proposition 8], Borwein and Borwein gave an example of a Lipschitz map (which is not pseudocontractive) with a unique fixed point for which the Mann sequence fails to converge; and in [6], Hicks and Kubicek gave an example of a discontinuous pseudocontraction with a unique fixed point for which the Mann iteration does not always converge. The problem for continuous pseudocontraction still remained open.

It is our purpose in this paper to resolve this problem by constructing an example of a Lipschitz pseudocontraction with a unique fixed point for which every nontrivial Mann sequence fails to converge. This settles the above open question.

\section{The EXAMPLE}

Let $X$ be the real Hilbert space $\mathbb{R}^{2}$ under the usual Euclidean inner product. If $x=(a, b) \in X$ we define $x^{\perp} \in X$ to be $(b,-a)$. Trivially, we have $\left\langle x, x^{\perp}\right\rangle=0$, $\left\|x^{\perp}\right\|=\|x\|,\left\langle x^{\perp}, y^{\perp}\right\rangle=\langle x, y\rangle,\left\|x^{\perp}-y^{\perp}\right\|=\|x-y\|$ and $\left\langle x^{\perp}, y\right\rangle+\left\langle x, y^{\perp}\right\rangle=0$ for all $x, y \in X$. We take our closed and bounded convex set $K$ to be the closed unit ball in $X$ and put $K_{1}=\left\{x \in X:\|x\| \leq \frac{1}{2}\right\}, K_{2}=\left\{x \in X: \frac{1}{2} \leq\|x\| \leq 1\right\}$. We 
define the map $T: K \longrightarrow K$ as follows:

$$
T x= \begin{cases}x+x^{\perp}, & \text { if } \quad x \in K_{1}, \\ \frac{x}{\|x\|}-x+x^{\perp}, & \text { if } \quad x \in K_{2} .\end{cases}
$$

We notice that, for $x \in K_{1} \cap K_{2}$, the two possible expressions for $T x$ coincide and that $T$ is continuous on both $K_{1}$ and $K_{2}$. Hence $T$ is continuous on all of $K$. We now show that $T$ is, in fact, Lipschitz: One easily shows that $\|T x-T y\|=\sqrt{2}\|x-y\|$ for $x, y \in K_{1}$. For $x, y \in K_{2}$, we have

$$
\begin{aligned}
\left\|\frac{x}{\|x\|}-\frac{y}{\|y\|}\right\|^{2} & =\frac{2}{\|x\|\|y\|}(\|x\|\|y\|-\langle x, y\rangle) \\
& =\frac{1}{\|x\|\|y\|}\left\{\|x-y\|^{2}-(\|x\|-\|y\|)^{2}\right\} \\
& \leq \frac{1}{\|x\|\|y\|} 2\|x-y\|^{2} \\
& \leq 8\|x-y\|^{2} .
\end{aligned}
$$

Hence, for $x, y \in K_{2}$, we have

$$
\|T x-T y\| \leq\left\|\frac{x}{\|x\|}-\frac{y}{\|y\|}\right\|+\|x-y\|+\left\|x^{\perp}-y^{\perp}\right\| \leq 5\|x-y\|,
$$

so that $T$ is Lipschitz on $K_{2}$. Now let $x$ and $y$ be in the interiors of $K_{1}$ and $K_{2}$ respectively. Then there exist $\lambda \in(0,1)$ and $z \in K_{1} \cap K_{2}$ for which $z=\lambda x+(1-\lambda) y$. Hence

$$
\begin{aligned}
\|T x-T y\| & \leq\|T x-T z\|+\|T z-T y\| \\
& \leq \sqrt{2}\|x-z\|+5\|z-y\| \\
& \leq 5\|x-z\|+5\|z-y\| \\
& =5\|x-y\| .
\end{aligned}
$$

Thus $\|T x-T y\| \leq 5\|x-y\|$ for all $x, y \in K$, as required.

The origin is clearly a fixed point of $T$. For $x \in K_{1},\|T x\|^{2}=2\|x\|^{2}$, and for $x \in K_{2},\|T x\|^{2}=1+2\|x\|^{2}-2\|x\|$. From these expressions and from the fact that $T x=x^{\perp} \neq x$ if $\|x\|=1$, it is easy to show that the origin is the only fixed point of $T$. We now show that no Mann iteration sequence for $T$ is convergent for any nonzero starting point:

First, we show that no such Mann sequence converges to the fixed point. Let $x \in K$ be such that $x \neq 0$. Then, in case $x \in K_{1}$, any Mann iterate of $x$ is actually further away from the fixed point of $T$ than $x$ is. This is because $\|(1-\lambda) x+\lambda T x\|^{2}=$ $\left(1+\lambda^{2}\right)\|x\|^{2}>\|x\|^{2}$ for $\lambda \in(0,1)$. If $x \in K_{2}$, then, for any $\lambda \in(0,1)$,

$$
\begin{aligned}
\|(1-\lambda) x+\lambda T x\|^{2} & =\left\|\left(\frac{\lambda}{\|x\|}+1-2 \lambda\right) x+\lambda x^{\perp}\right\|^{2} \\
& =\left[\left(\frac{\lambda}{\|x\|}+1-2 \lambda\right)^{2}+\lambda^{2}\right]\|x\|^{2} \\
& >0 .
\end{aligned}
$$

More generally, it is easy to see that for the recursion formula (1.2), if $x_{0} \in K_{1}$, then $\left\|x_{n+1}\right\|>\left\|x_{n}\right\|$ for all integers $n \geq 0$, and if $x_{0} \in K_{2}$, then $\left\|x_{n+1}\right\| \geq \frac{\sqrt{2}}{2}\left\|x_{n}\right\|$ for all integers $n \geq 0$. We therefore conclude that, in addition, any Mann iterate of any nonzero vector in $K$ is itself nonzero. Thus any Mann sequence $\left\{x_{n}\right\}$, starting from 
a nonzero vector, must be infinite. For such a sequence to converge to the origin, $x_{n}$ would have to lie in the neighbourhood $K_{1}$ of the origin for all $n>N_{0}$, for some real $N_{0}$. This is not possible because, as already established for $K_{1},\left\|x_{n}\right\|<\left\|x_{n+1}\right\|$ for all $n>N_{0}$.

We now show that no Mann sequence converges to $x \neq 0$. We do this in the form of a general lemma.

Lemma 2.1. Let $M$ be a nonempty, closed and convex subset of a real Banach space $E$ and let $S: M \rightarrow M$ be any continuous function. If a Mann sequence for $S$ is norm convergent, then the corresponding limit is a fixed point for $S$.

Proof. Let $x_{n}$ be a Mann sequence in $M$ for $S$, as defined in the recursion formula (1.2). Assume, for proof by contradiction, that the sequence converges, in norm, to $x$ in $M$, where $S x \neq x$. For each $n \in \mathbb{N}$, put $\epsilon_{n}=x_{n}-S x_{n}-x+S x$. Since $S$ is continuous, the sequence $\epsilon_{n}$ converges to 0 . Pick $p \in \mathbb{N}$ such that, if $m \geq p$ and $n \geq p$, then $\left\|\epsilon_{n}\right\|<\frac{\|x-S x\|}{3}$ and $\left\|x_{n}-x_{m}\right\|<\frac{\|x-S x\|}{3}$. Pick any positive integer $q$ such that $\sum_{n=p}^{p+q} c_{n} \geq 1$. We have that

$$
\begin{aligned}
\left\|x_{p}-x_{p+q+1}\right\| & =\left\|\sum_{n=p}^{p+q}\left(x_{n}-x_{n+1}\right)\right\| \\
& =\left\|\sum_{n=p}^{p+q} c_{n}\left(x-S x+\epsilon_{n}\right)\right\| \\
& \geq\left\|\sum_{n=p}^{p+q} c_{n}(x-S x)\right\|-\left\|\sum_{n=p}^{p+q} c_{n} \epsilon_{n}\right\| \\
& \geq \sum_{n=p}^{p+q} c_{n}\left(\|x-S x\|-\frac{\|x-S x\|}{3}\right) \geq \frac{2\|x-S x\|}{3} .
\end{aligned}
$$

The contradiction proves the result.

We now show that $T$ is a pseudocontraction. First, we note that we may put $j(x)=x$, since $X$ is Hilbert. For $x, y \in K$, put $\Gamma(x ; y)=\|x-y\|^{2}-\langle T x-T y, x-y\rangle$ and, if $x$ and $y$ are both nonzero, put $\lambda(x ; y)=\frac{\langle x, y\rangle}{\|x\|\|y\|}$. Hence, to show that $T$ is a pseudo-contraction, we need to prove that $\Gamma(x ; y) \geq 0$ for all $x, y \in K$. We only need examine the following three cases:

1. $x, y \in K_{1}: \quad$ An easy computation shows that $\langle T x-T y, x-y\rangle=\|x-y\|^{2}$ so that $\Gamma(x ; y)=0$; thus we are home and dry for this case.

2. $x, y \in K_{2}$ : Again, a straightforward calculation shows that

$$
\begin{aligned}
\langle T x-T y, x-y\rangle & =\|x\|-\|x\|^{2}+\|y\|-\|y\|^{2}+\langle x, y\rangle\left(2-\frac{1}{\|x\|}-\frac{1}{\|y\|}\right) \\
& =\|x\|-\|x\|^{2}+\|y\|-\|y\|^{2}+\lambda(x ; y)(2\|x\|\|y\|-\|x\|-\|y\|) .
\end{aligned}
$$

Hence $\Gamma(x ; y)=2\|x\|^{2}+2\|y\|^{2}-\|x\|-\|y\|-\lambda(x ; y)(4\|x\|\|y\|-\|x\|-\|y\|)$. It is not hard to establish that $(4\|x\|\|y\|-\|x\|-\|y\|) \geq 0$ for all $x, y \in K_{2}$. Hence, for fixed $\|x\|$ and $\|y\|, \Gamma(x ; y)$ has a minimum when $\lambda(x ; y)=1$. This 
minimum is therefore $2\|x\|^{2}+2\|y\|^{2}-4\|x\|\|y\|=2(\|x\|-\|y\|)^{2}$. Again, we have that $\Gamma(x ; y) \geq 0$ for all $x, y \in K_{2}$ as required.

3. $x \in K_{1}, y \in K_{2}$ : We have

$$
\langle T x-T y, x-y\rangle=\|x\|^{2}+\|y\|-\|y\|^{2}-\lambda(x ; y)\|x\| .
$$

Hence $\Gamma(x ; y)=2\|y\|^{2}-\|y\|+(\|x\|-2\|x\|\|y\|) \lambda(x ; y)$. Since $\|x\|-2\|x\|\|y\| \leq 0$ for $x \in K_{1}$ and $y \in K_{2}, \Gamma(x ; y)$ has its minimum, for fixed $\|x\|$ and $\|y\|$ when $\lambda(x ; y)=1$. We conclude that

$$
\begin{aligned}
\Gamma(x ; y) & \geq 2\|y\|^{2}-\|y\|+\|x\|-2\|x\|\|y\| \\
& =(\|y\|-\|x\|)(2\|y\|-1) \\
& \geq 0 \text { for all } x \in K_{1}, y \in K_{2} .
\end{aligned}
$$

This completes the proof.

Remark 2.2. In [11, Qihou proved that if $K$ is a compact convex subset of a Hilbert space and $T: K \rightarrow K$ is a continuous pseudocontractive map with a finite number of fixed points, then the Ishikawa iteration sequence defined by (1.3) converges strongly to a fixed point of $T$. Consequently, while the Mann sequence does not converge to the fixed point of $T$ in our example, the Ishikawa sequence does.

\section{REFERENCES}

1. F.E. Browder, Nonexpansive nonlinear operators in a Banach space, Proc. Nat. Acad. Sci. U.S.A. 54 (1965), 1041-1044. MR 32:4574

2. D. Borwein and J.M. Borwein, Fixed point iterations for real functions, J. Math. Anal. Appl. 157 (1991), 112-126. MR 92e:65065

3. S. S. Chang, The Mann and Ishikawa iterative approximation of solutions to variational inclusions with accretive type mappings, Comput. Math. Appl. 37 (1999), no.9, 17-24. MR 2000g:47065

4. C. E. Chidume, Global iteration schemes for strongly pseudo-contractive maps, Proc. Amer. Math. Soc. (1998) Vol. 126, No 9, 2641-2649. MR 98k:47108

5. D. Göhde, Zum Prinzip der kontraktiven Abbildungen, Math. Nachr. 30 (1965), 251-258. MR 32:8129

6. T. L. Hicks and J. R. Kubicek, On the Mann iteration process in Hilbert space, J. Math. Anal. Appl. 59 (1979), 498-504. MR 58:23802

7. S. Ishikawa, Fixed points by a new iteration method, Proc. Amer. Math. Soc. 44(1) (1974), 147-150. MR 49:1243

8. W. A. Kirk, A fixed point theorem for mappings which do not increase distance, Amer. Math. Monthly 72 (1965), 1004-1006. MR 32:6436

9. M. A. Krasnoselski, Two observations about the method of succesive approximations, Uspehi Math. Nauk 10 (1955), no. 1, 123-127. (Russian) MR 16:833a

10. W. R. Mann, Mean value methods in iteration, Proc. Amer. Math. Soc. 4 (1953), 506-510. MR 14:988f

11. Liu Qihou, The convergence theorems of the sequence of Ishikawa iterates for hemicontractive mappings, J. Math. Anal. Appl. 148 (1990), 55-62. MR 92b:47094

12. H. Schaefer, Uber die Methode sukzessiver Approximationen, Jber. Deutsch. Math. Verein. 59 (1957), 131-140. MR 18:811g

The Abdus Salam International Centre for Theoretical Physics, 34100 Trieste, Italy

E-mail address: chidume@ictp.trieste.it

Department of Physics, University of Zimbabwe, Harare, Zimbabwe 